J. Math. Kyoto Univ. (JMKYAZ)

14-1 (1974) 73-92

\title{
Perturbation of drift-type for Lévy processes
}

\author{
Hiroshi TaNaKa, Masaaki Tsuchiya \\ and Shinzo Watanabe
}

(Received 6, Feb. 1973)

\section{§1. Introduction.}

Given a temporally homogeneous Lévy process $l(t)$ and a real valued bounded Borel function $a(x)$ on $R^{1}$, we consider the following stochastic equation:

$$
d x(t)=d l(t)+a(x(t)) d t
$$

The precise meaning of this equation will be given in $\S 2$. When $l(t)$ is a symmetric Cauchy process, Tsuchiya [1] proved that the above equation has a unique solution for each initial value $x \in R^{1}$ if $a(x)$ is small enough in the supremum norm. The purpose of this paper is to give a sufficient condition in terms of the characteristic function of $l(1)$ in order that the equation (1.1) has a unique solution for any bounded continuous function $a(x)$. In [1] only the uniqueness of the probability law of a solution process $x(\cdot)$ was considered, but in fact as will be remarked in $\S 2$, this "weak uniqueness" is equivalent to the apparently stronger "pathwise uniqueness". When $l(t)$ is a symmetric Cauchy process, our result (Theorem 3.1) implies, as a special case, that the equation (1.1) has a unique solution for any bounded continuous $a(x)$. But, for general bounded Borel $a(x)$ the problem is still open. Also, Theorem 3.1 does not cover the case of symmetric Lévy process with exponent $\alpha<1$; in this case, however, some uniqueness and non-uniqueness results will be obtained in connection with the modulus of continuity of $a(x)$ (Theorem 3.2). Fianlly in $\S 4$, some 
remarks will be given for the equation (1.1) in case when $a(x)$ has a jump-discontinuity.

\section{§2. Solutions of stochastic equation (1.1).}

A Lévy process we consider in this paper is a 1-dimensional process $l(t)$ with stationary independent increments whose sample paths are right continuous and have limits from the left; also it is assumed that $l(0)=0$. The function $\psi(\xi)$ denotes the logarithm of the characteristic function of $l(1)$; its canonical form is

$$
\psi(\xi)=i m \xi-\frac{v}{2} \xi^{2}+\int_{-\infty}^{\infty}\left(e^{i \xi^{k}}-1-\frac{i \xi u}{1+u^{2}}\right) n(d u), \xi \in R^{1},
$$

where $m \in R^{1}, v \geqq 0, \int_{-\infty}^{\infty} \frac{u^{2}}{1+u^{2}} n(d u)<\infty$. The notation $\left(\Omega, \dot{F}, P ; \mathscr{F}_{t}\right)$ stands for a probability space $(\Omega, \mathscr{F}, P)$ endowed with an increasing family of sub- $\sigma$-fields $\mathscr{F}_{t}$ of $\mathscr{F}$. We now indroduce the following definitions.

Definition 1. By a solution of (1.1) we mean a stochastic process $x(t)$ defined over a suitable probability space $\left(\Omega, \mathscr{F}, P ; \mathscr{F}_{t}\right)$ satisfying the following three conditions.

(i) There exists a Lévy process $l(t)$ such that

$$
E\left\{e^{i \xi(l(t)-l(s))} \mid \mathscr{F}_{s}\right\}=\exp ((t-s) \psi(\xi)), \quad 0 \leqq s<t, \quad \xi \in R^{1} .
$$

(ii) $x(t)$ and $l(t)$ are $\mathscr{F}_{t}$-measurable for each $t \geqq 0$.

(iii) $x(t)=x(0)+l(t)+\int_{0}^{t} a(x(s)) d s$ holds with probability 1 .

The process $l(t)$ is called the underlying Lévy process for the solution $x(t)$.

Definition 2. We say that the pathwise uniqueness holds for (1.1) if any two solutions, defined over a common probability space $(\Omega$, $\mathscr{F}, P ; \mathscr{F}_{t}$ ) with a common initial value and with a common underlying Lévy process, are equal with probability 1.

Definition 3. We say that the uniqueness in law holds for (1.1) 
if any two solutions with a common initial distribution induce the same probability law on the space of all real valued right continuous functions on $[0, \infty)$ having limits from the left.

Exactly in the same way as in [2], we can prove thta the pathwise uniqueness implies the uniqueness in law. Moreover, in the present case the converse is also true as will be proved in the following proposition, and so we shall omit "pathwise" or "in law" thereafter.

Proposition 1.1. The uniqueness in law implies the pathwise uniqueness.

Proof. Let $x_{1}(t)$ and $x_{2}(t)$ be two solutions of (1.1) defined over a common probability space $\left(\Omega, \mathscr{F}, P ; \mathscr{F}_{t}\right)$ with a common initial value and a common underlying Lévy process $l(t)$. Then, by the following lemma $x(t)=\min \left(x_{1}(t), x_{2}(t)\right)$ is also a solution of (1.1), and hence the uniqueness in law ensures that the probability distribution of $x(t)$ is equal to that of $x_{1}(t)$. This obviously implies that $x_{1}(t) \leqq$ $x_{2}(t) \quad(a . s$.$) and hence x_{2}(t) \leqq x_{1}(t) \quad(a . s$.$) by symmetry, yielding the$ result.

Lemma. If $x_{1}(t)$ and $x_{2}(t)$ are solutions of

$$
x(t)=x(0)+l(t)+\int_{0}^{t} a(x(s)) d s
$$

then $x(t)=\min \left(x_{1}(t), x_{2}(t)\right)$ is also a solution of (2.2).

Proof. For each $\omega$ in a certain subset $\Omega_{0}$ of full measure, the function $x_{i}(t, \omega)$ of $t \geqq 0$ satisfies (2.2) $(i=1,2)$. Fixing such an $\omega$ and then writing $x_{i}(t), l(t)$ for $x_{i}(t, \omega), l(t, \omega)$, we set $y_{i}(t)=x_{i}(t)-l(t)$ $(i=1,2), a(t, x)=a(l(t)+x)$. Then, that the $x_{i}(t)$ satisfies (2.2) is equivalent to

$$
y_{i}(t)=x+\int_{0}^{t} a\left(s, y_{i}(s)\right) d s, \quad i=1,2 .
$$

We now set $y(t)=\min \left(y_{1}(t), y_{2}(t)\right)$, and claim that $y(t)$ satisfies 


$$
y(t)=x+\int_{0}^{t} a(s, y(s)) d s .
$$

Since the open set $I=\left\{t>0: y_{1}(t)>y_{2}(t)\right\}$ can be expressed as the disjoint sum of open intervals $\left(s_{k}, t_{k}\right)$, we have for any $C^{1}$-function $\phi$ with support contained in $(0, \infty)$

$$
\begin{aligned}
& -\int_{0}^{\infty} \phi^{\prime}(t) y(t) d t=-\int_{[0, \infty)-I} \phi^{\prime}(t) y_{1}(t) d t-\int_{I} \phi^{\prime}(t) y_{2}(t) d t \\
= & -\int_{[0, \infty)-I} \phi^{\prime}(t)\left(x+\int_{0}^{t} a\left(s, y_{1}(s)\right) d s\right) d t-\int_{I} \phi^{\prime}(t)\left(x+\int_{0}^{t} a\left(s, y_{2}(s)\right) d s\right) d t \\
= & -\int_{0}^{\infty} \phi^{\prime}(t) \int_{0}^{t} a\left(s, y_{1}(s)\right) d s d t+\int_{I} \phi^{\prime}(t) \int_{0}^{t} a\left(s, y_{1}(s)\right) d s d t \\
= & -\int_{I}^{\infty} \phi(t) a\left(t, y_{1}(t)\right) d t+\int_{0}^{t} a\left(s, y_{2}(s)\right) d s d t \\
& -\sum_{k}\left[\phi(t) \int_{0}^{t} a\left(s, y_{1}(s)\right) d s\right]_{s_{k}}^{t_{k}}-\int_{I} \phi(t) a\left(t, y_{1}(t)\right) d t \\
= & \left.\int_{0}^{\infty} \phi(t) a(t, y(t)) d t+\sum_{k}\left[\phi(t)\left(y_{1}(t)-x\right)\right]_{s_{k}}^{t_{k}}-\sum_{k}[\phi(t)) d s\right]_{s_{k}}^{t_{k}}+\int_{I} \phi(t) a\left(t, y_{2}(t)\right) d t \\
= & \int_{0}^{\infty} \phi(t) a(t, y(t)) d t .
\end{aligned}
$$

Thus $y(t)$ satisfies (2.3), and this implies that $x(t)$ is a solution of (2.2).

In the rest of this section, we construct the maximum and minimum solutions in case $a(x)$ is continuous. Suppose that $a(x)$ is bounded and continuous, and choose a decreasing sequence $\left\{a_{n}(x)\right\}$ of uniformly bounded and Lipschitz continuous functions such that $a_{n}(x)$ decreases to $a(x)$ as $n \uparrow \infty$. Then, as is well known, the equation

$$
x(t, \omega)=x+l(t ; \omega)+\int_{0}^{t} a_{n}(x(s, \omega)) d s
$$

has a unique solution $x_{n}(t, x, \omega)$ for each $\omega \in \Omega$ and $x \in R^{1}$, where 
$l(t)$ is a Lévy process defined over a probability space $(\Omega, \mathscr{F}, P)$. It is easy to verify that $x_{n}(t, x, \omega)$ decreases to some limit as $n \uparrow \infty$, and that this limit $\bar{x}(t, x, \omega)$ is the biggest among solutions of (1.1) with initial value $x$ and with underlying Lévy process $l(t)$; the $\bar{x}(t, x$, $\omega)$ is called the maximum solution of (1.1) with underlying Lévy process $l(t)$. The minimum solution $\underline{x}(t, x, \omega)$ can be constructed similarly.

Sometimes it is convenient to consider the standard (path space) representation of the underlying Lévy process $l(t)$. Denote by $W$ the space of right continuous functions on $[0, \infty)$ having limits from the left and vanishing at $t=0$, by $\mathscr{M}_{t}$ the smallest $\sigma$-field that makes $\{w(s): 0 \leqq s \leqq t\}$ measurable, and put $\mathscr{M}=\vee \mathscr{M}_{t}$. For each $t \geqq 0$ let $\theta_{t}$ denote the mapping from $W$ into itself defined by $\left(\theta_{t} w\right)(s)=w(t+s)-$ $w(t)$. Let $\gamma$ be the mapping from $\Omega$ into $W$ defined by $\gamma \omega=w$ where $w(t)=l(t, \omega)$. Then, $\gamma$ is $\mathscr{F} / \mathscr{M}$-measurable and induces a probability measure $\mathscr{P}$ over $(W, \mathscr{M})$ with respect to which the coordinate process $w(t)$ is a Lévy process equivalent in law to the process $l(t)$. Denote by $x^{\sharp}(t, x, w)$ the maximum solution of

$$
x(t)=x+w(t)+\int_{0}^{t} a(x(s)) d s .
$$

Then the followings are easily proved.

$1^{\circ}$. For each fixed $t \geqq 0, x^{\sharp}(t, x, w)$ is $\mathscr{B}\left(R^{1}\right) \times \mathscr{M}_{t}$-measurable.

$2^{\circ} . x^{\sharp}(t+s, x, w)=x^{\sharp}\left(s, x^{\sharp}(t, x, w), \theta_{t} w\right)$,

$3^{\circ}$. The maximum solution $\bar{x}(t, x, \omega)$ with underlying Lévy process

$l(t)$ is expressed as $\bar{x}(t, x, \omega)=x^{\sharp}(t, x, \gamma \omega)$.

$4^{\circ}$. The family of random functions $\left\{\bar{x}(t, x, \omega), x \in R^{1}\right\}$ defines a temporally homogeneous Markov process on $R^{1}$; this Markov process is a Hunt process.

The minimum solutions have similar properties.

\section{§3. Uniqueness and nonuniqueness.}

First we consider the case of bounded continuous $a(x)$ and give a sufficient condition for the uniqueness of solutions of (1.1). 
Theorem 3.1. Suppose that $a(x)$ is bounded and continuous, and that the function $\psi(\xi)$ associated with a given Lévy process satisfies

$$
\frac{1}{\mathscr{R} \psi(\xi)}=0\left(\frac{1}{|\xi|}\right), \xi \longrightarrow \pm \infty
$$

Then, there exists a unique solution of (1.1).

Proof. Since the "existence" part is obvious (the maximum and minimum solutions exist), it is sufficient to prove the uniqueness. The assumption (3.1) implies that

$$
\frac{1}{|\mathscr{R} \psi(\xi)|} \leqq \frac{K}{|\xi|} \quad \text { for all } \xi \in R^{1} \text { such that }|\xi|>\xi_{0}
$$

where $\xi_{0}$ and $K$ are positive constants fixed throughout the proof. Let $p_{t}(x)$ be the probability density with characteristic function $\exp \{t \psi$ $(-\xi)\}$, and set $g_{\lambda}(x)=\int_{0}^{\infty} e^{-\lambda t} p_{t}(x) d t, \lambda>0$. Then $g_{\lambda} \in L^{1}\left(R^{1}\right) \cap L^{2}\left(R^{1}\right)$. Moreover, if we set

$$
\hat{g}(\xi)=\frac{1}{\sqrt{2 \pi}} \int_{-\infty}^{\infty} e^{i \xi x} g_{\lambda}(x) d x=\frac{1}{\sqrt{2 \pi}} \cdot \frac{1}{\lambda-\psi(-\xi)}
$$

then by (3.2) we have

$$
\left|\xi \hat{g}_{\lambda}(\xi)\right| \leqq \frac{K}{\sqrt{2 \pi}} \quad \text { for } \lambda>\frac{\xi_{0}}{K} .
$$

Let $\bar{x}(t)$ be the maximum solution of (1.1) with initial value $x$ and with underlying Lévy process $l(t)$, and introduce the Green operator $K_{\lambda}$ :

$$
\left(K_{\lambda} f\right)(x)=\int_{0}^{\infty} e^{-\lambda t} E\{f(\bar{x}(t))\} d t, \quad \lambda>0, f \in L^{2}\left(R^{1}\right) .
$$

We first condiser the following special case: (3.4) $\left\{\begin{array}{l}\text { The support of the Lévy measure } n(\cdot) \text { of } l(t) \text { is contained } \\ \text { in some finite interval }[-h, h] \text {. }\end{array}\right.$ 
Lemma 3.1. If $a(x) \in C^{2}, a(x), a^{\prime}(x), a^{\prime \prime}(x)$ are bounded and if the supremum norm $\|a\|_{\infty}<\frac{\sqrt{2 \pi}}{K}$, then under the above assumption (3.4), we have

$$
K_{\lambda} f=G_{\lambda}\left(I-a D G_{\lambda}\right)^{-1} f, f \in L^{2}, \lambda>\frac{\xi_{0}}{K},
$$

where $G_{\lambda}$ is the convolution operator: $\left(G_{\lambda} f\right)(x)=\left(f_{*} g_{\lambda}\right)(x), \lambda>0$ and $D=\frac{d}{d x}$.

Proof. The smoothness assumption on $a(x)$ implies that (1.1) with initial value $x$ and with underlying Lévy process $l(t)$ has a unique solution which we denote by $x(t, x)$ to stress the initial position. Setting $y(t)=y(t, x)=\frac{\partial x(t, x)}{\partial x}, z(t)=z(t, x)=\frac{\partial^{2} x(t, x)}{\partial x^{2}}$ and then writing the differential equations for $y(t)$ and $z(t)$, we can see that $|y(t)| \leqq \exp \left(c_{1} t\right)$ and $|z(t)| \leqq c_{2} \exp \left(3 c_{1} t\right)$ where $c_{1}=\left\|a^{\prime}\right\|_{\infty}, c_{2}=\left\|a^{\prime \prime}\right\|_{\infty}$. Therefore if $f$ is in $C_{0}^{2}$, the space of $C^{2}$-functions with compact supports, then

$$
\begin{aligned}
& \left\|D K_{\lambda} f\right\|_{\infty} \leqq\left\|f^{\prime}\right\|_{\infty} \int_{0}^{\infty} e^{-\lambda t} e^{c_{1} t} d t \\
& \left\|D^{2} K_{\lambda} f\right\|_{\infty} \leqq\left\|f^{\prime \prime}\right\|_{\infty} \int_{+}^{\infty} e^{-\lambda t} e^{2 c_{1} t} d t+c_{2}\left\|f^{\prime}\right\|_{\infty} \int_{0}^{\infty} e^{-\lambda t} e^{3 c_{1} t} d t .
\end{aligned}
$$

Thus $D K_{\lambda} f$ and $D^{2} K_{\lambda} f$ exist for $\lambda>3 c_{1}$ and are bounded continuous, and hence $u=K_{\lambda} f$ satisfies

$$
(\lambda-A-a D) u=f, \quad f \in C_{0}^{2}, \lambda>3 c_{1}
$$

where $A$ is the infinitesimal generator of $l(t)$ viewed as a Markov process. We next claim that $D u \in L^{2}\left(R^{1}\right)$. For this we use the assumption (3.4). Take a finite open interval $I$ of length $>h$ such that $\operatorname{supp}(f) \subset I$, and then choose a finite open interval $J$ such that $J \supset \bar{I}$. Then

$$
\begin{aligned}
|D u| & \leqq E\left\{\int_{0}^{\infty} e^{-\lambda t}\left|f^{\prime}(x(t))\right| e^{c_{1} t} d t\right\} \\
& \leqq\left\|f^{\prime}\right\|_{\infty}\left(K_{\lambda_{0}} g\right)(x), \quad \lambda_{0}=\lambda-c_{1}>2 c_{1}, g=\chi_{I} .
\end{aligned}
$$


Since $D u$ is a bounded function, we are sufficed to show that $|D u(x)|$ is bounded from above by a square integrable function outside $J$. For $x \notin J$, put $T(x)=\inf \{t>0: x(t, x) \in I\}$. Then we have

$$
|D u(x)| \leqq\left\|f^{\prime}\right\|_{\infty} \cdot \lambda_{0}^{-1} E\left\{e^{-\lambda_{0} T(x)}\right\} .
$$

In the case when $x$ lies entirely in the right-side of $J$, we set $\widetilde{T}(x)=$ $\inf \{t>0: x+\tilde{l}(t) \in I\}$ where $\tilde{l}(t)=l(t)-\|a\|_{\infty} \cdot t$. Then by the assumption (3.4) $\tilde{T}(x) \leqq T(x)$. On the other hand, if we set

$$
v(x)=\left(\widetilde{G}_{\lambda_{0}} h\right)(x) \equiv E\left\{{ }_{0}^{\infty} e^{-\lambda_{0} t} h(\tilde{l}(t)+x) d t,\right\} \quad h=\chi_{J},
$$

then

$$
v(x) \geqq c E\left\{e^{-\lambda \tilde{T}(x)}\right\}, c=\min _{y \in \bar{I}}\left(\tilde{G}_{\lambda_{0}} h\right)(y)>0 .
$$

Noting that the assumption (3.1) implies that $v \in L^{2}\left(R^{1}\right)$ and using (3.6) and $E\{\exp (-\lambda T(x))\} \leqq E\{\exp (-\lambda \widetilde{T}(x))\} \leqq c^{-1} v(x)$, we obtain $\int_{0}^{\infty}|D u(x)|^{2}<\infty$. By a similar argument, we have $\int_{-\infty}^{0}|D u(x)|^{2}<\infty$, and thus $D u \in L^{2}\left(R^{1}\right)$.

Once we have proved $D u \in L^{2}\left(R^{1}\right)$, then by (3.5) we have

$$
\begin{aligned}
u & =G_{\lambda}(f+a D u) \\
& =G_{\lambda} \sum_{k=0}^{n}\left(a D G_{\lambda}\right)^{k} f+G_{\lambda}\left(a D G_{\lambda}\right)^{n} a D u, \quad \lambda>3 c_{1} .
\end{aligned}
$$

By (3.3) and the assumption $\|a\|_{\infty}<\frac{\sqrt{2 \pi}}{K}$, we have for $f \in L^{2}\left(R^{1}\right)$

$$
\left\|a D G_{\lambda} f\right\|_{2} \leqq \rho\|f\|_{2}, \lambda>\frac{\xi_{0}}{K} \quad\left(\rho=\|a\|_{\infty} \cdot \frac{K}{\sqrt{2 \pi}}<1\right),
$$

and hence by (3.7)

$$
K_{\lambda} f=G_{\lambda}\left(I-a D G_{\lambda}\right)^{-1} f, \quad \lambda>\max \left(3 c_{1}, \frac{\xi_{0}}{K}\right), f \in C_{0}^{2} .
$$

Since both sides of the above exist and are analytic (in $\lambda$ ) for $\lambda>\frac{\xi_{0}}{K}$ 
and $f \in L^{2}\left(R^{1}\right)$, (3.8) holds for all $\lambda>\frac{\xi_{0}}{K}, f \in L^{2}\left(R^{1}\right)$, completing the proof of the lemma.

We now proceed to the proof of the uniqueness for (1.1) under the assumption (3.4)

Case I. $a$ is continuous and $\|a\|_{\infty}<\frac{\sqrt{2 \pi}}{K}$. Choose a sequence of functions $\left\{a_{n}(x)\right\}$ such that

(i) each $a_{n}(x)$ is a $C^{2}$-function, $a_{n}^{\prime}(x), a_{n}^{\prime \prime}(x)$ are bounded, and $\sup _{n}\left\|a_{n}\right\|_{\infty}<\frac{\sqrt{2 \pi}}{K}$.

(ii) $a_{n}(x)$ decreases to $a(x)$ as $n \uparrow \infty$.

Denote by $K_{\lambda, n}$ the Green operator of the Markov process defined by the family of unique solutions of $d x(t)=d l+a_{n}(x(t)) d t$, and by $K_{\lambda}$ the Green operator corresponding to the maximum solutions of (1.1). Then $\left(K_{\lambda, n} f\right)(x)$ coverges as $n \rightarrow \infty$ to $\left(K_{\lambda} f\right)(x)$ for any bounded continuous function $f$. But then by Lemma 3.1 for any continuous function $f$ in $L^{2}\left(R^{1}\right)$ and each $x$

$$
\begin{aligned}
K_{\lambda} f(x)=\lim _{n \rightarrow \infty} K_{\lambda, n} f(x) & =\lim _{n \rightarrow \infty} G_{\lambda}\left(I-a_{n} D G_{\lambda}\right)^{-1} f(x) \\
& =G_{\lambda}\left(I-a D G_{\lambda}\right)^{-1} f(x), \lambda>\frac{\xi_{0}}{K}
\end{aligned}
$$

By similar arguments to the above, we see that the Green operator corresponding to the minimum solutions of (1.1) has the form $G_{\lambda}(I-$ $\left.a D G_{\lambda}\right)^{-1}$ on $L^{2}\left(R^{1}\right)$, and hence the maximum and minimum solutions are identical, proving the uniqueness for (1.1).

Case II. $a(x)$ is bounded continuous.

For each fixed $X_{0} \in R^{1}$, we shooce $\varepsilon>0$ so that $\left|a(x)-a\left(x_{0}\right)\right|<$ $\sqrt{2 \pi} / K$ holds for $\left|x-x_{0}\right| \leqq \varepsilon$, and set $\tilde{a}(x)=a(x)-a\left(x_{0}\right)$. Also take a continuous function $a_{0}(x)$ such that it coincides with $\tilde{a}(x)$ for $\left|x-x_{0}\right|$ $\leqq \varepsilon$ and $\left\|a_{0}\right\|_{\infty}<\sqrt{2 \pi} / K$. Then (1.1) is equivalent to 


$$
d x(t)=d \tilde{l}+\tilde{a}(x(t)) d t
$$

where $\tilde{l}(t)=l(t)+a\left(x_{0}\right) \cdot t$, and the maximum (minimum) solution $\bar{x}\left(t, x_{0}\right)$ $\left(\underline{x}\left(t, x_{0}\right)\right)$ of the latter equation with initial value $x_{0}$ coincides with the corresponding solution of $d x(t)=d \tilde{l}+a_{0}(x(t)) d t$ for small values of $t$. Since the real part of the function $\tilde{\psi}(\alpha)$ associated with $\gamma(t)$ is equal to $\mathscr{R} \psi(\alpha)$, the result of case $\mathrm{I}$ is applicable to $d x(t)=d \tilde{l}+a_{0}(x(t)) d t$ and hence the uniqueness for this equation holds. Thus we have proved $\bar{x}\left(t, x_{0}\right)=\underline{x}\left(t, x_{0}\right)$ for small values of $t$. Next we set

$$
\begin{aligned}
& \sigma(\omega)=\sigma\left(x_{0}, \omega\right)=\inf \left\{t>0 ; \bar{x}\left(t, x_{0}, \omega\right)>\underline{x}\left(t, x_{0}, \omega\right)\right\}, \\
& \tau(w)=\tau\left(x_{0}, w\right)=\inf \left\{t>0 ; x^{\sharp}\left(t, x_{0}, w\right)>x_{b}\left(t, x_{0}, w\right\}\right),
\end{aligned}
$$

where $x^{\#}$ and $x_{b}$ are the maximum and minimum solutions of (1.1) with underlying Lévy process $\{W, w(t), \mathscr{P}\}$. Then by $3^{\circ}$ of $\S 2 P\left[\sigma\left(x_{0}\right.\right.$, $\omega)<t]=\mathscr{P}\left[\tau\left(x_{0}, w\right)<t\right]$. Therefore, for our present purpose we are sufficed to show that $\mathscr{P}\left[\tau\left(x_{0}, w\right)<\infty\right]=0$. This is verified as follows. By the meaning of $\tau\left(x_{0}, w\right)$ and $2^{\circ}$ of $\S 2, \tau\left(x_{0}, w\right)<\infty$ implies that $\tau\left(x^{\#}\left(\tau\left(x_{0}, w\right), x_{0}, w\right), \theta_{\tau\left(x_{0}, w\right)} w\right)=0$, and hence

$$
\begin{aligned}
\mathscr{P}\left[\tau\left(x_{0}, w\right)<\infty\right] & =\mathscr{P}\left[\tau\left(x^{\sharp}\left(\tau\left(x_{0}, w\right), \theta_{\tau\left(x_{0}, w\right)} w\right)=0, \tau\left(x_{0}, w\right)<\infty\right]\right. \\
& =\mathscr{P}\left[f\left(x^{\sharp}\left(\tau\left(x_{0}, w\right)\right) ; \tau\left(x_{0}, w\right)<\infty\right],\right.
\end{aligned}
$$

where $f(x)=\mathscr{P}[\tau(x, w)=0]$; but $f(x)=0$ as we have proved before, and so $\mathscr{P}\left[\tau\left(x_{0}, w\right)<\infty\right]=0$.

Finally we have to remove the restriction (3.4). In general $l(t)$ is expressed as the sum $l_{1}(t)+l_{2}(t)$ where $l_{1}(t)$ and $l_{2}(t)$ are two independent Lévy processes and the support of the Lévy measure of $l_{1}(t)$ is compact (the assumption (3.4) is satisfied for $l_{1}(t)$ ) and the sample paths of $l_{2}(t)$ are step functions. Under this situation, it is clear that the following two statements are equivalent:

(a) The uniqueness holds for $d x(t)=d l+a(x(t)) d t$.

(b) The uniqueness holds for $d x(t)=d l_{1}+a(x(t)) d t$.

Now the uniqueness for (1.1) follows from this remark.

Remark 1 (A sufficient condition for the uniqueness in case of 
bounded Borel measurable $a(x)$ ). If (3.1) is replaced by the stronger condition: $(\mathscr{R} \psi(\xi))^{-1}=o\left(|\xi|^{-1}\right)$ as $|\xi| \rightarrow \infty$, then there exists a unique solution of (1.1) for any bounded Borel measurable function $a(x)$. The proof is similar to that of Tsuchiya [1].

When $l(t)$ is a symmetric stable process with exponemt $\alpha<1$, the condition (3.1) is not satisfied. In this case, results in the uniqueness problem for the equation (1.1) depend upon the modulus of continuity of $a(x)$, as will be seen in the following theorem.

\section{Theorem 3.2.}

If the underling Lévy process $l(t)$ is a symmetric stable process with exponent $\alpha<1$, we have the following results.

(i) If $a(x)$ is a bounded non-decreasing function and satisfies

$$
|a(x)-a(y)| \leqq K|x-y|^{\beta}
$$

for some positive constants $K$ and $\beta$ with $1-\alpha<\beta \leqq 1$, then the uniqueness holds for the equation (1.1).

(ii) If $a(x)$ is bounded continuous and equal to sign $x|x|^{\beta}$ in some neighborhood of $x=0$ for some positive constant $\beta<1-\alpha$, then the uniqueness does not hold for the equation (1.1) under the initial condition $x(0)=0$.

Before giving the proof, we prepare two lemmas.

Lemma 3.2. Let $a(x)$ be bounded continuous and non-decreasing, and $\bar{x}(t)(\underline{x}(t))$ be the maximum(minimum) solution of (1.1). Let $y(t)$ be a right continuous process satisfying

$$
y(t) \leqq \bar{x}(0)+l(t)+\int_{0}^{t} a(y(s)) d s, \quad 0 \leqq t \leqq h,
$$

where $h=h(\omega)>0$. Then $y(t) \leqq \bar{x}(t), 0 \leqq t \leqq h$. If " $\leqq$ " and $\bar{x}(0)$ in (3.9) are replaced by " $\geqq$ " and $\underline{x}(0)$ respectively, then the conclusion is $y(t) \leqq \underline{x}(t), 0 \leqq t \leqq h$.

Proof. For $\varepsilon>0$, set $a_{\varepsilon}(x)=a(x)+\varepsilon$, and let $x_{\varepsilon}(t)$ be a solution of $x(t)=\bar{x}(0)+l(t)+\int_{0}^{t} a_{\varepsilon}(x(s)) d s$. Then 
84 Hiroshi Tanaka, Masaaki Tsuchiya and Shinzo Watanabe

$$
x_{\varepsilon}(t)-y(t) \geqq \int_{0}^{t}\left\{a_{\varepsilon}\left(x_{\varepsilon}(s)\right)-a(y(s))\right\} d s, \quad 0 \leqq t \leqq h .
$$

Now suppose $y(t)>x_{\varepsilon}(t)$ for some $t \in[0, h]$, and let $t_{0}$ be the infimum of $t$ for which $x_{\varepsilon}(t)<y(t)$. Then, obviously $t_{0}>0$ and

$$
\int_{0}^{t_{0}}\left\{a_{\varepsilon}\left(x_{\varepsilon}(s)\right)-a(y(s))\right\} d s \geqq \int_{0}^{t_{0}}\left\{a_{\varepsilon}(y(s))-a(y(s))\right\} d s \geqq \varepsilon t_{0},
$$

and hence $x_{\varepsilon}\left(t_{0}\right)-y\left(t_{0}\right) \geqq \varepsilon t_{0}$. But this contradicts the right continuity of $x_{\varepsilon}(t)-y(t)$. Therefore, $x_{\varepsilon}(t) \geqq y(t), 0 \leqq t \leqq h$, and making $\varepsilon \downarrow 0$ we obtain the result.

Lemma 3.3. Suppose $a(x)$ satisfies the condition (i) of Theorem 3.2 and vanishes at $x=x_{0}$, and let $x(t)$ be any solution of (1.1) with initial condition $x(0)=x_{0}$. Then for any $\varepsilon>0$

$$
\lim _{t \rightarrow 0} t^{-\frac{1}{\alpha}+\varepsilon}\left|x(t)-x_{0}\right|=0
$$

holds almost surely.

Proof. We may assume $x_{0}=0$ and also $\varepsilon<\frac{1}{\alpha}-1$. By a theorem of Hinčin [3], we have

$$
P\left\{\lim _{t \downarrow 0} t^{-\frac{1}{\alpha}+\varepsilon}|l(t)|=0\right\}=1
$$

for each $\varepsilon>0$, and hence there exists $t_{\varepsilon}=t_{\varepsilon}(\omega)>0$ such that $|l(t)| \leqq t^{\frac{1}{\alpha}-\varepsilon}$ for $o \leqq t \leqq t_{\varepsilon}$, almost surely. Therefore, for $0 \leqq t \leqq t_{\varepsilon},|x(t)|$ is dominated by the maximum solution $m(t)$ of

$$
m(t)=t^{\frac{1}{\alpha}-\varepsilon}+K \int_{0}^{t}|m(s)|^{\beta} d s .
$$

On the other hand, $m(t)$ can be constructed as follows. Set

$$
m_{0}(t)=1, m_{n}(t)=t^{\frac{1}{\alpha}-\varepsilon}+K \int_{0}^{t}\left|m_{n-1}(s)\right|^{\beta} d s \quad(n \geqq 1) .
$$

Then, $m_{n}(t)$ decreases to $m(t)$ as $n \uparrow \infty$ for $0 \leqq t \leqq t_{0}$ where $t_{0}$ is some 
constant with $0<t_{0}<1$. Since $\beta>1-\alpha$, we see by induction that

$$
m_{n}(t) \leqq \text { const. } t^{\frac{1}{\alpha}-\varepsilon}+\text { const. } t^{1+\beta+\cdots+\beta^{n-1}}, 0 \leqq t \leqq t_{0},
$$

and hence $m_{n}(t) \leqq$ const. $t^{\frac{1}{\alpha-\varepsilon}}$ for sufficently large $n$. Therefore we have

$$
|x(t)| \leqq m_{n}(t) \leqq \text { const. } t^{\frac{1}{\alpha}-\varepsilon}, 0 \leqq t \leqq t_{0} \wedge t_{\varepsilon}(\omega),
$$

and this implies (3.10), since $\varepsilon>0$ is arbitrary.

Proof of Theorem 3.2 (i) We may deal with the standard (path space) representation of the underlying Lévy process $l(t)$ as in the end of $\S 2$. Denote by $\bar{G}_{\lambda}\left(\underline{G}_{\lambda}\right)$ the Green operator of the Markov process defined by the maximum solutions $x^{\sharp}(t, x, w), x \in R^{1}$ (minimum solutions $\left.x_{b}(t, x, w), x \in R^{1}\right)$ of (1.1). Then it is enough to prove $\left(\bar{G}_{\lambda} f\right)$ $(x) \leqq\left(\underline{G}_{\lambda} f\right)(x)$ for any bounded continuous and non-decreasing function $f$, since $\left(\underline{G}_{\lambda} f\right)(x) \leqq\left(\bar{G}_{\lambda} f\right)(x)$ is obvious. Fixing $x \in R^{1}$, we first consider the case $a(x) \leqq 0$. We set

$$
T_{n}(w)=\inf \left\{t>0: x^{\sharp}(t, x, w)<x-\frac{1}{n}\right\},
$$

and claim that

$$
\mathscr{P}\left\{T_{n}(w) \downarrow 0 \quad \text { as } n \rightarrow \infty\right\}=1 .
$$

If $a(x)<0$, this is obvious; if $a(x)=0$, we notice that

$$
\left|\int_{0}^{t} a\left(x^{\sharp}(s, x, w)\right) d s\right| \leqq K \int_{0}^{t}\left|x^{\sharp}(s, x, w)-x\right|^{\beta} d s \leqq \text { const. } t^{\left(\frac{1}{\alpha}-\varepsilon\right) \beta+1}
$$

for all sufficiently small $t>0$ by Lemma 3.3. Since $\beta>1-\alpha$, we can choose $\varepsilon>0$ so small that $\left(\frac{1}{\alpha}-\varepsilon\right) \beta+1>\frac{1}{\alpha}$. Then we have $\int_{0}^{t} a\left(x^{\sharp}\right) d s=$ $o\left(t^{\frac{1}{\alpha}}\right)$ as $t \downarrow 0$. This combined with a result of Hinčin [3]:

$$
\mathscr{P}\left\{\varlimsup_{t \rightarrow 0} t^{-\frac{1}{\alpha}}|l(t)|=\infty\right\}=1
$$

implies (3.11). Now we take a function $f$ with the properties indicated 
before, and write for $\lambda>0$

$$
\begin{aligned}
\left(\bar{G}_{\lambda} f\right)(x)= & \mathscr{E}\left\{\int_{0}^{T_{n}} e^{-\lambda t} f\left(x^{\sharp}(t, x, w)\right) d t\right\} \\
& +\mathscr{E}\left\{\int_{T_{n}}^{\infty} e^{-\lambda t} f\left(x^{\sharp}(t, x, w) d t ; T_{n}<\infty\right\}=I_{1}+I_{2} .\right.
\end{aligned}
$$

Then

$$
\begin{aligned}
& \left|I_{1}\right| \leqq\|f\|_{\infty} \cdot \mathscr{E}\left\{\frac{1-e^{-\lambda T_{n}}}{\lambda}\right\} \longrightarrow 0 \quad \text { as } n \longrightarrow \infty \\
& I_{2}=\mathscr{E}\left\{e^{-\lambda T_{n}} \int_{0}^{\infty} e^{-\lambda t} f\left(x^{\sharp}\left(t, x^{\sharp}\left(T_{n}, x, w\right), \theta_{T_{n}} w\right)\right) d t ; T_{n}<\infty\right\} .
\end{aligned}
$$

On the other hand, by the non-decreasing property of $a(\cdot)$ we have $x^{\sharp}(t, y, w)<x_{b}(t, x, w)$ for $t \geqq 0$ provided $y<x$, and hence

$$
x^{\sharp}\left(t, x^{\sharp}\left(T_{n}, x, w\right), \theta_{T_{n}} w\right)<x_{b}\left(t, x, \theta_{T_{n}} w\right),
$$

because $x^{\sharp}\left(T_{n}, x, w\right)<x$. Therefore

$$
I_{2} \leqq \mathscr{E}\left\{e^{-\lambda T_{n}} \int_{0}^{\infty} e^{-\lambda t} f\left(x_{b}\left(t, x, \theta_{T_{n}} w\right)\right) d t ; T_{n}<\infty\right\}=\mathscr{E}\left\{e^{-\lambda T_{n}}\right\}\left(G_{\lambda} f\right)(x),
$$

and hence by making $n \uparrow \infty$ in (3.12) we obtain $\left(\bar{G}_{\lambda} f\right)(x) \leqq\left(G_{\lambda} f\right)(x)$. Finally it remains to treat the case $a(x)>0$, but this is done by exchanging the role of the maximum and minimum solutions in the above proof.

(ii) is proved by showing that the maximum solution $\bar{x}(t)$ and minimum solution $\underline{x}(t)$ of (1.1) with initial value 0 are different. By the assumption for $a(x)$, there exists $\varepsilon_{0}>0$ such that $a(x)=\operatorname{sign} x|x|^{\beta}$ for $|x| \leqq \varepsilon_{0}$. We introduce a bounded continuous and non-decreasing function $\tilde{a}(x)$ such that $\tilde{a}(x)=a(x)$ for $|x| \leqq \varepsilon_{0}$, and denote by $\tilde{x}(t)$ $(x(t))$ the maximum (minimum) solution of (1.1) with initial value 0 when $a(x)$ is replaced by $\tilde{a}(x)$. Then, obviously $\bar{x}(t)=\tilde{x}(t)$ and $x(t)=$ $x(t)$ for all sufficiently small $t$ with probability 1 , and hence it is enough to show that $\underset{\sim}{x}(t)<\tilde{x}(t)$ for all sufficiently small $t>0$ with probability 1. For $c>0$ let $\Omega_{c}$ be the set of $\omega$ for which there exists $h=h(\omega)>0$ 
such that $|l(t, \omega)| \leqq c t^{\delta}$ for all $t \in[0, h]$, where $\delta=\frac{1}{1-\beta}\left(<\frac{1}{\alpha}\right)$. Then Hinčin' result tells us $P\left\{\Omega_{c}\right\}=1$. We now choose $c>0$ and $d>0$ so that $\delta^{-1} d^{\beta}-c \geqq d$, and then $h_{1}>0$ so that $h_{1}^{\delta} d \leqq \varepsilon_{0}$; we define $y(t, \omega)$ for $\omega \in \Omega_{c}$ by

$$
y(t, \omega)= \begin{cases}t^{\delta} d & \text { for } 0 \leqq t \leqq h(\omega) \wedge h_{1} \\ \left(h(\omega) \wedge h_{1}\right)^{\delta} d & \text { for } t \geqq h(\omega) \wedge h_{1} .\end{cases}
$$

Then, for $\omega \in \Omega_{c}$ and $0 \leqq t \leqq h(\omega) \wedge h_{1}$ we have

$$
\begin{aligned}
l(t, \omega)+\int_{0}^{t} a(y(s, \omega)) d s=l(t, \omega)+d^{\beta} \int_{0}^{t} s^{\beta \delta} d s \\
=l(t, \omega)+d^{\beta} \frac{t^{\delta}}{\delta} \geqq-|l(t, \omega)|+\frac{d^{\beta}}{\delta} \cdot t^{\delta} \\
\geqq-c t^{\delta}+\frac{d^{\beta}}{\delta} \cdot t^{\delta}=\left(\frac{d^{\beta}}{\delta}-c\right) t^{\delta} \geqq t^{\delta} d=y(t, \omega)
\end{aligned}
$$

and hence $\tilde{x}(t) \geqq y(t)=t^{\delta} d$ for $0 \leqq t \leqq h(\omega) \wedge h_{1}$ by Lemma 3.2. Similarly, $x(t) \leqq-t^{\delta} d$ for $0 \leqq t \leqq h(\omega) \wedge h_{1}$. Therefore, $x(t)<\tilde{x}(t)$ for all sufficiently small $t>0$ almost surely.

\section{§4. Remarks to the case of discontinuous $a(x)$.}

In this section we consider the case in which $a(x)$ has a jumpdiscontinuity at $x=x_{0}$; for simplicity we assume $x_{0}=0$ and that there exists a constant $K$ such that

$$
|a(x)-a(y)| \leqq K|x-y| \quad \text { for } x, y \in R^{1} \quad \text { with } x \cdot y>0 \text {. }
$$

We set $a(0)=0$ and $a_{ \pm}=\lim _{x \rightarrow 0 \pm} a(x)$. Given a right continuous function $l(t)$ with limits from the left and $l(0)=0$, we consider the equation

$$
x(t)=x+l(t)+\int_{0}^{t} a(x(s)) d s+\int_{0}^{t} \delta(s) d s
$$

under the additional condition 
(4. 1b) $\delta(s)=0$ if $x(s) \neq 0$, and $a_{-} \leqq \delta(s) \leqq a_{+}$or $a_{+} \leqq \delta(s) \leqq a_{-}$.

The uniqueness theorem for this equation has different aspect according to $a_{-}<a_{+}$or $a_{+}<a_{-}$.

Case I: $a_{+}<a_{-}$.

Proposition 4.1. For each $x \in R^{1}$, the uniqueness for (4.1) holds.

Proof. Let $x_{1}(t)$ and $x_{2}(t)$ be two solutions of (4.1) with initial value $x$, that is,

$$
\begin{aligned}
& x_{1}(t)=x+l(t)+\int_{0}^{t} a\left(x_{1}(s)\right) d s+\int_{0}^{t} \delta_{1}(s) d s \\
& x_{2}(t)=x+l(t)+\int_{0}^{t} a\left(x_{2}(s)\right) d s+\int_{0}^{t} \delta_{2}(s) d s .
\end{aligned}
$$

Put $y(t)=x_{1}(t)-x_{2}(t)$ and $\sigma(t)=a\left(x_{1}(t)\right)-a\left(x_{2}(t)\right)$. Then $y(t)=\int_{0}^{t} \sigma(s) d s$. It is easy to show that $y(t)>0$ implies $\sigma(t) \leqq K y(t)$. Now suppose $y(t)$ $>0$ for some $t>0$ and set $t_{0}=\inf \{s \leqq t: y(r)>0$ for all $r \in(s, t]\}$. Then $y(t)=\int_{t_{0}}^{t} \sigma(s) d s \leqq K \int_{t_{0}}^{t} y(s) d s$ and this yields $y(t)=0$, a contradiction. Therefore $y(t) \leqq 0$ and hence $x_{1}(t) \leqq x_{2}(t)$. Similarly we have $x_{1}(t) \geqq x_{2}(t)$ and so $x_{1}(t)=x_{2}(t)$.

Proposition 4.2. For each initial value $x$ there exists a solution $x(t, x)$ of (4.1), and $x(t, x)$ is continuous in $x$ for each $t \geqq 0$.

Proof. Define a Lipshitz continuous function $a_{n}(x)$ by

$$
a(x)= \begin{cases}a(x) & \text { for } x<0 \text { or } x>\frac{1}{n} \\ n\left\{a\left(\frac{1}{n}\right)-a_{-}\right\} x+a_{-} & \text {for } 0 \leqq x \leqq \frac{1}{n},\end{cases}
$$

and denote by $x_{n}(t)=x_{n}(t, x)$ the unique solution of

$$
x(t)=x+l(t)+\int_{0}^{t} a_{n}(x(s)) d s .
$$


Since the sequence of functions $\left\{a_{n}(x)\right\}$ is non-increasing for all sufficiently large $n, x_{n}(t, x) \geqq x_{n+1}(t, x)$ for all sufficiently large $\mathrm{n}$, and hence we can define $\bar{x}(t, x)=\lim _{n \rightarrow \infty} x_{n}(t, x)$; it is right continuous in $x$ (upper semi-continuous in this case) and satisfies

$$
\lim _{n \rightarrow \infty} \int_{0}^{t} a_{n}\left(x_{n}(s)\right) \chi(\bar{x}(s)) d s=\int_{0}^{t} a(\bar{x}(s)) d s
$$

where $\chi$ is the indicator function of $\{0\}^{c}$. If we set

$$
\varphi_{n}(t)=\int_{0}^{t} a_{n}\left(x_{n}(s)\right) \chi_{\{0\}}(\bar{x}(s)) d s
$$

then $\varphi(t)=\lim _{n \rightarrow \infty} \varphi_{n}(t)$ exists and

$$
\bar{x}(t)=x+l(t)+\int_{0}^{t} a(\bar{x}(s)) d s+\varphi(t)
$$

Since $\left|\varphi\left(t_{2}\right)-\varphi\left(t_{1}\right)\right| \leqq\|a\|_{\infty}\left|t_{2}-t_{1}\right|$, there exists a function $\delta(s)$ such that $\varphi(t)=\int_{0}^{t} \delta(s) d s$ and $|\delta(s)| \leqq\|a\|_{\infty} ;$ moreover we can choose $\delta(s)$ so that $\bar{x}(s) \neq 0$ implies $\delta(s)=0$ and $a_{+} \leqq \delta(s) \leqq a_{-}$for all $s \geqq 0$. Therefore, $\bar{x}(t)$ is a solution of (4.1). Approximating the function $a(x)$ by increasing sequence of Lipshitz continuous functions, we can construct a solution $\underline{x}(t)$ of $(4.1)$ such that $\underline{x}(t, x)$ is left continuous in $x$ for each $t \geqq 0$. By Proposition 4.1, $\underline{x}(t)=\bar{x}(t)$ and this proves Proposition 4.2.

We now replace $l(t)$ by a sample path of a time homogneous Lévy process. If we take the standard representation $(W, l(t, w), \mathscr{M}$, $\left.\mathscr{M}_{t}, \mathscr{P}\right)$ of the Lévy process, then the unique solution $x(t, x, w)$ of $(4.1)$ with $l(t)=l(t, w)$ satisfies $x(t+s, x, w)=x\left(s, x(t, x, w), \theta_{t} w\right)$ and hence we have the following theorem.

Theorem 4.1. When $l(t)$ is a time homogeneous Lévy process, the family $\left\{x(t, x, w), x \in R^{1}\right\}$ of the unique solutions of (4.1) defines a Markov process whose semigroup maps $C_{b}^{*}$ into itself; (4.1) is

*) $C_{b}$ is the space of bounded continuous real valued functions on $R^{1}$. 
written as follows:

$$
x(t)=x+l(t)+\int_{0}^{t} a(x(s)) d s+a_{0} \int_{0}^{t} \chi_{\{0\}}(x(s)) d s,
$$

where $a_{0}$ is a constant such that $a_{+} \leqq a_{0} \leqq a_{-}$.

Proof. The only task we have to do is to show that $x(t)$ satisfies (4.2). Set

$$
\begin{aligned}
& y_{n}=x\left(t+\frac{1}{n}, x, w\right)-x(t, x, w)-\int_{0}^{\frac{1}{n}} a\left(x\left(s, x(t, x, w), \theta_{t} w\right)\right) d s \\
& \quad-l\left(\frac{1}{n}, \theta_{t} w\right) \\
& z_{n}=x\left(\frac{1}{n}, x, w\right)-x-\int_{0}^{\frac{1}{n}} a(x(s, x, w)) d s-l\left(\frac{1}{n}, w\right) \\
& a(x, w)=\varlimsup_{n \leftarrow \infty} n z_{n} .
\end{aligned}
$$

By the equation (4.1a) $\delta(t)=\varlimsup_{n \rightarrow \infty} n y_{n}=a\left(x(t, x, w), \theta_{t} w\right)$. On the other hand, $x(t, x, w)$ is $\mathscr{M}_{t}$-measurable and hence $a(x, w)$ is $\mathscr{M}_{0+}$-measurable for each $x$. Therefore by Blumenthal's $0-1$ law $a(x, w)$ is a constant (a.s.) for each $x$, and it is clear that the constant is zero for $x \neq 0$. We denote by $a_{0}$ the constant for $x=0$; obviously $a_{+} \leqq a_{0} \leqq a_{-}$. For each $x$ and $t$ there exists a sequence $\left\{\Delta_{n}\right\}$ of divisions of $[0, t]$, say, $\Delta_{n}: 0=t_{n, 0}<t_{n, 1}<\cdots<t_{n, k(n)}=t$ such that

$$
\begin{aligned}
& \int_{0}^{t} a\left(x(s, x, w), \theta_{s} w\right) d s=\lim _{n \rightarrow \infty} \sum_{j=0}^{k(n)-1} a\left(x\left(t_{n, j}, x, w\right), 0_{t_{n, j}} w\right)\left(t_{n, j+1}-t_{n, j}\right) \\
& \int_{0}^{t} \chi_{\{0\}}(x(s, x, w)) d s=\lim _{n \rightarrow \infty} \sum_{j=0}^{k(n)-1} \chi_{\{0\}}\left(x\left(t_{n, j}, x, w\right)\right)\left(t_{n, j+1}-t_{n, j}\right)
\end{aligned}
$$

with probability 1. Since $x\left(t_{n, j}, x, w\right)$ is independent of $\theta_{t_{n, j}} w$ and $l\left(t, \theta_{t_{n}, j} w\right)$ is a Lévy process equivalent in law to $l(t)$, we have

$$
\mathscr{P}\left\{a\left(x\left(t_{n, j}, x, w\right), \theta_{t_{n, j}} w\right)=a_{0} \chi_{\{0\}}\left(x\left(t_{n, j}, x, w\right)\right)\right\}=1
$$

and hence we have $\int_{0}^{t} \delta(s) d s=a_{0} \int_{0}^{t} \chi_{\{0\}}(x(s, x, w)) d s$, proving (4.2). 
Corollary. If $l(t)$ is a symmetric Lévy process and $a(x)$ is an odd function, then the equation (1.1) has a unique solution.

Proof. If $x(t)$ is the solution of (4.2) with initial value 0 , then $y(t)=-x(t)$ and $l^{-}(t, w)=-l(t, w)$ satisfy

$$
y(t)=l^{-}(t, w)+\int_{0}^{t} a(y(s)) d s-a_{0} \int_{0}^{t} \chi_{\{0\}}(y(s)) d s
$$

Since $l^{-}(t, w)$ is a Lévy process equivalent in law to $l(t, w)$, we can take $a_{0}$ so that $-a_{0}=a_{0}$, that is, $a_{0}=0$.

Case II: $a_{-}<a_{+}$. By a method similar to the proof of Proposition 4.2 we can construct two solutions $\bar{x}(t, x)$ and $\underline{x}(t, x)$ of (4.1); for each $t \geqq 0, \bar{x}(t, x)(\underline{x}(t, x))$ is a non-decreasing and right (left continuous function of $x$, and $\underline{x}(t, x) \leqq \bar{x}(t, x)$. The present case turns out to the case I under time reversion, and this fact will now be used to prove the following proposition.

Proposition 4.3. Let $x(t)$ and $y(t)$ be any solutions of (4.1) with initial values $x$ and $y$, reprectively. Then,

$$
x(t)<y(t) \text { whenever } x<y ;
$$

moreover, $\bar{x}(t, x)$ and $\underline{x}(t, x)$ are the maximum and minimum solutions of (4.1) respectively.

Proof. Suppose there exists $t_{0}>0$ such that $x\left(t_{0}\right)=y\left(t_{0}\right)$, and set for $0 \leqq t \leqq t_{0}$

$$
\begin{aligned}
& \hat{x}(t)=-x\left(t_{0}-t\right), \quad l(t)=l\left(t_{0}\right)-l\left(t_{0}-t\right), \\
& \hat{a}(x)=a(-x), \quad \hat{\delta}(t)=\delta\left(t_{0}-t\right) .
\end{aligned}
$$

Then we have

$$
\hat{x}(t)=-x\left(t_{0}\right)+\hat{l}(t)+\int_{0}^{t} \hat{a}(x(s)) d s+\int_{0}^{t} \hat{\delta}(s) d s,
$$

which is the equation of Case I only apart from the left continuity 
of $\hat{l}(t)$; the situation is the same for $y(t)$, and hence by the uniqueness result of Proposition 4.1 we have $\hat{x}(t)=\hat{y}(t)$ for $0 \leqq t \leqq t_{0}$ with obvious notation, contradicting $\hat{x}\left(t_{0}\right)=-x>-y=\hat{y}\left(t_{0}\right)$.

By virture of Proposition 4.3 (especially (4.3)) we obtain the following theorem; the proof is much the same as that of (i) of Theorem 3.2 and so is omitted.

Theorem 4.2. Let $a_{-}<a_{+}$and take for $l(t)$ in (4.1) sample paths of a Lévy process. If the Lévy process satisfies

$$
\mathscr{P}\left\{\varlimsup_{t \rightarrow 0} \frac{|l(t)|}{t}=\infty\right\}=1
$$

then $\bar{x}(t, x)=x(t, x)$ almost surely for each $x \in R^{1}$.

The corollary to Theorem 4.1 can also be adapted to Case II under the condition (4.4).

$$
\begin{aligned}
& \text { Hiroshima University (H. Tanaka) } \\
& \text { Kanazawa University (M. Tsuchiya) } \\
& \text { KYoto University (S. Watanabe) }
\end{aligned}
$$

\section{References}

[1] M. Tsuchiya, On a small drift of Cauchy process, J. Math. Kyoto Univ., 10 (1970), 475-492.

[2] T. Yamada and S. Watanabe, On the uniqueness of solution of stochastic differential equations. J. Math. Kyoto Univ., 11 (1971), 155-167.

[3] А.Я. ХИНЧИН, Две теоремы о стохастических процессах с однотипнымн приращениями. Математический Сборник Т. 3 (45), (1938) 577-584. 